\title{
Magnetic anisotropy in Li-phosphates and origin of magnetoelectricity in $\mathrm{LiNiPO}_{4}$
}

\author{
Kunihiko Yamauchi and Silvia Picozz $*$ \\ Consiglio Nazionale delle Ricerche - Istituto Nazionale di Fisica della Materia (CNR-INFM), \\ CASTI Regional Lab., 67100 L'Aquila, Italy
}

(Dated: November 26, 2018)

\begin{abstract}
Li-based phosphates are paradigmatic materials for magnetoelectricity. By means of firstprinciples calculations, we elucidate the microscopic origin of spin anisotropy and of magnetoelectric effects in $\mathrm{LiNiPO}_{4}$. The comparison with $\mathrm{LiCoPO}_{4}$ reveals that $\mathrm{Co}-d^{7}$ and $\mathrm{Ni}-d^{8}$ electronic clouds show distinct orbital shapes, which in turn result in an opposite trend of the local spin anisotropy with respect to the surrounding $\mathrm{O}_{6}$ cages. Due to magnetic anisotropy, the Ni-based phosphate shows a peculiar "angled-cross" spin ground-state, which is responsible for magnetoelectricity. In this respect, we show that, under a magnetic field $H_{x}$, an electronic polarization $P_{z}$ arises, with an estimated linear magneto-electric coefficient in good agreement with experiments.
\end{abstract}

PACS numbers: Valid PACS appear here

Olivine phosphates $\mathrm{LiCoPO}_{4}$ and $\mathrm{LiNiPO}_{4}$ are attracting large interests, due to their peculiar magnetoelectric (ME) effect [i.e. the control of ferroelectric (magnetic) properties via a magnetic (electric) field] as well as their application for electrodes in rechargeable Li batteries [1, 2]. Recently, ferrotoroidic domains and antiferromagnetic domains have been independently observed in $\mathrm{LiCoPO}_{4}$ by using second harmonic generation. [3] The toroidal moment, generated by a vortex of magnetic moments, is considered as source of a novel ferroic order, closely related to ME effects [4, 5] as well as to multiferroicity [6] (i.e. coexistence of long-range magnetic and dipolar orders). In fact, $\mathrm{LiCoPO}_{4}$ shows a nonzero linear ME coefficient, $\alpha_{x y}$ and $\alpha_{y x}$, at low temperature, consistent with the toroidal moment $T$ nearly parallel to $z$ axis [5], whereas $\mathrm{LiNiPO}_{4}$ shows $\alpha_{x z}$ and $\alpha_{z x}$ 7]. Although magnetoelectricity has been macroscopically investigated by means of Landau theory [8, 9], its microscopic origin has not been clarified yet nor first principles calculations aimed at investigating $\mathrm{ME}$ effects in phosphates exist in the literature. $\mathrm{In} \mathrm{LiCoPO}_{4}$, antiferromagnetic (AFM) spins were found to be along the $b$ axis 10 or uniformly rotated from this axis by $4.6^{\circ} 8$. In $\mathrm{LiNiPO}_{4}$, it was proposed the collinear AFM spins to lie along the $c$ axis [11], but recently a non-collinear structure in a "spin-cross" style [9, 12] was suggested, in order to explain the butterfly shape of the ME hystheresis curve 13.

In this letter, within Density-functional theory (DFT) we investigate the magnetic anisotropy for Li-phosphates $\mathrm{LiTMPO}_{4}(\mathrm{TM}=\mathrm{Ni}, \mathrm{Co})$ and we are able to correlate the orbital degree of freedom with the calculated anisotropy and spin-configuration. The discussion on magnetic anisotropy is particularly important for $\mathrm{LiNiPO}_{4}$, where we find, as magnetic ground-state, a peculiar spin-cross configuration that leads to magnetoelectricity; finally, we

*Electronic address: silvia.picozzi@aquila.infn.it clarify the microscopic origin of ME effects, our calculated ME coefficient being in quantitative agreement with experiments.

Methodology and structural details. DFT simulations were performed using the VASP code 14] and the PAW pseudopotentials [15] within the GGA $+U$ formalism [16] ( $U=5 \mathrm{eV}$ and $J=0 \mathrm{eV}$ for $\mathrm{Ni} d$-states). Other values of $U=2$ and $8 \mathrm{eV}$ were also tested. The cut-off energy for the plane-wave expansion of the wave-functions was set to $400 \mathrm{eV}$ and a k-point shell of $(2,4,4)$ was used for the Brillouin zone integration. Lattice parameters were fixed as experimentally observed [17, 18]. The internal atomic coordinates were fully optimized in a (fully-compensated) AFM configuration, keeping $S_{1}=S_{2}=-S_{3}=-S_{4}$ configuration. In the orthorhombic Pnma $\left(D_{16}^{2 h}\right)$ paramagnetic space group, these four $\mathrm{Co} / \mathrm{Ni}$ sites are related by eight symmetry operations; all the rotations and mirror reflections accompany translations, so that the $\mathrm{Co} / \mathrm{Ni}$ sites are slightly deviated from high-symmetry positions. Optimized coordinates of the four $\mathrm{Co} / \mathrm{Ni}$ ions are: $\mathrm{Co} 1 / \mathrm{Ni} 1(1 / 4+\epsilon, 1 / 4,-\delta), \mathrm{Co} 2 / \mathrm{Ni} 2(3 / 4+\epsilon, 1 / 4$, $1 / 2+\delta), \mathrm{Co} 3 / \mathrm{Ni} 3(3 / 4-\epsilon, 3 / 4, \delta), \mathrm{Co} 4 / \mathrm{Ni} 4(1 / 4-\epsilon, 3 / 4$, $1 / 2-\delta)$, where $\epsilon=0.0268$ and $\delta=0.0207$ for Co, $\epsilon=0.0249$ and $\delta=0.0152$ for Ni. The spin-orbit coupling (SOC) term was computed self-consistently inside each atomic sphere, with a radius of $1 \AA$. The electronic polarization $P$ was calculated using the Berry phase method[19].

Magnetic anisotropy: $\mathrm{LiCoPO}_{4}$ vs $\mathrm{LiNiPO}_{4}$. As shown in Fig 1, each Co ion is surrounded by highly distorted oxygen octahedra, so that the partially filled $t_{2 g}$ shell shows a dumbbell-shaped charge distribution, axially elongated along the $\mathbf{n}_{\mathbf{i}}$ direction (along $\mathrm{m} 1-\mathrm{m} 2$ O-Co$\mathrm{O}$ bonds). This peculiar shape is expected to induce a strong local magnetic anisotropy via the SOC term. On the other hand, in $\mathrm{LiNiPO}_{4}$, the Ni- $d^{8}$ orbital shows a more isotropic sphere-like shape.

Both the global and local magnetic anisotropy were investigated by rotating four spins simultaneously, keeping a collinear AFM coupling. The global magnetic anisotropic energy (MAE) was calculated by differ- 

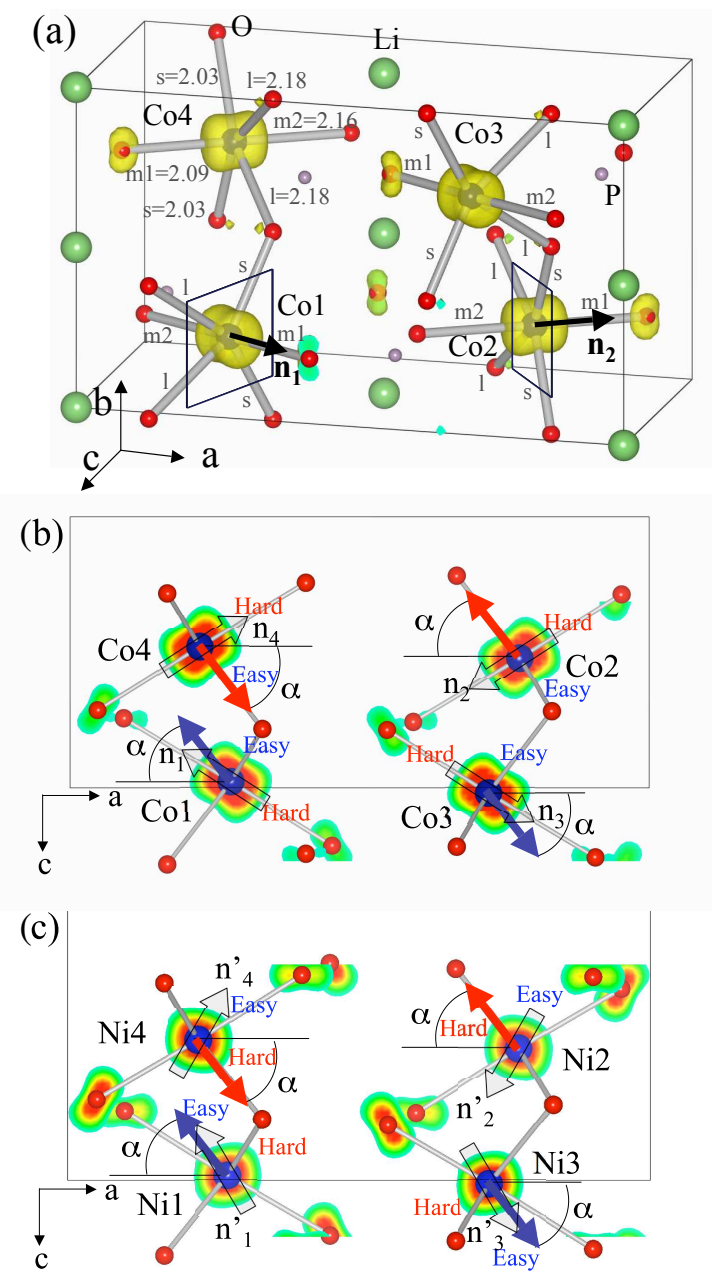

FIG. 1: (a) Isosurface of charge density of minority-spin Co$t_{2 g}$ states (within an energy range up to $1 \mathrm{eV}$ below $E_{F}$ ). CoO bond lenghts $(\AA)$ are reported and denoted as $l, m 1, m 2, s$. Easy planes for Co local spins and the corresponding normal vectors (hard axis) $\mathbf{n}_{\mathbf{i}}$ are also shown at Co1 and Co2 sites. (b) Section of charge density of Co $t_{2 g}$ electrons and magnetic easy and hard axis in the $a c$ plane in $\mathrm{LiCoPO}_{4}$. (c) Same as (b) for $\mathrm{LiNiPO}_{4}$. Unfilled large arrows show hard $\left(n_{i}\right)$ and easy $\left(n_{i}^{\prime}\right)$ axis in ac plane for Co and $\mathrm{Ni}$ spins respectively.

ences in the total energy with different spin-orientations, whereas the local anisotropic energy was evaluated as proportional to the expectation value of the SOC energy: $E_{\mathrm{SOC}}=\left\langle\frac{1}{c^{2}} \frac{1}{r} \frac{d V}{d r} l \cdot s\right\rangle[20$ ] (integrated in each atomic sphere).

Fig. 2 (a) and (b) shows the MAE of Co spins in the $a c$ and $a b$ planes, respectively. The global easy axis is the $b$ direction, in agreement with the experimental suggestion that spins should be aligned along the $b$ axis (possibly with a slight rotation). The MAE is rather high (more than 10 times larger than the orbitally-ordered Mn spins in $\mathrm{TbMnO}_{3}[21]$ ). As shown by the spin angle dependence of $E_{S O C}$ (cfr Fig. 2(a)), the easy/hard axial direction depends on each Co site. $E_{t o t}$ can be fitted by a conventional quadratic anisotropic term for $S_{i}$ spins:
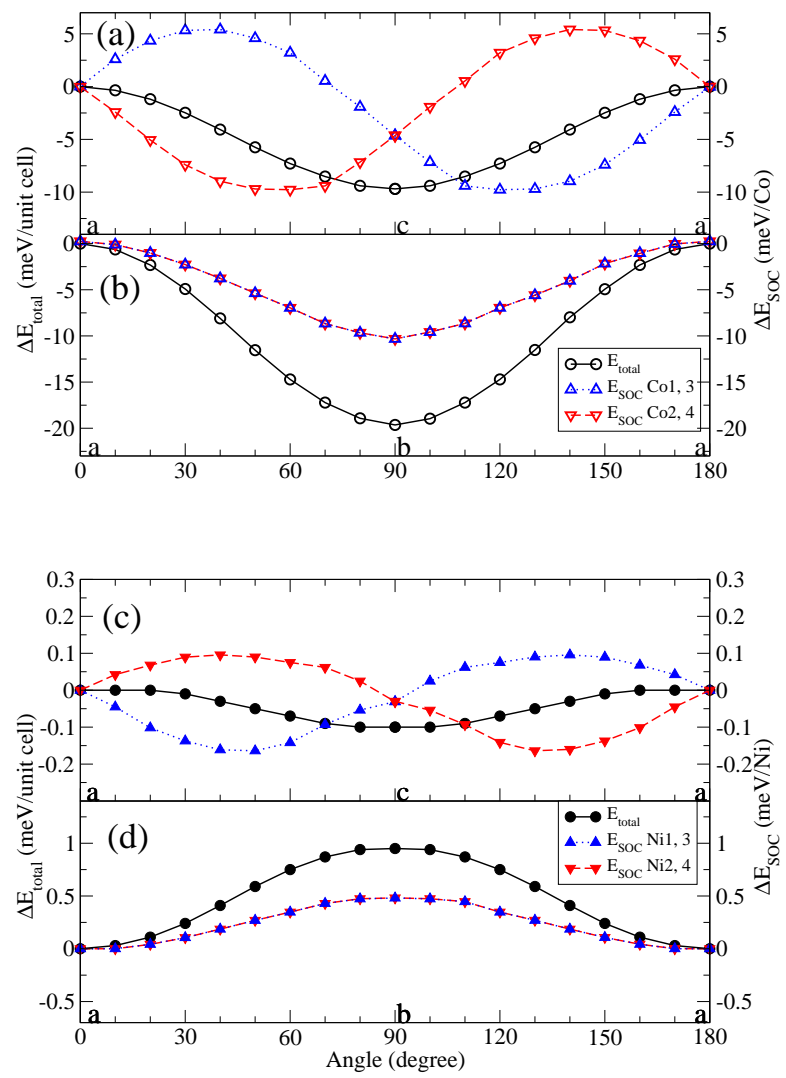

FIG. 2: Total energy $\Delta E_{\text {total }}$ (black solid line) and $\Delta E_{S O C}$ (dashed colored lines) at each Co site vs the direction of collinear Co spins (a) in $a c$ plane and (b) in $a b$ plane. Corresponding curves for $\mathrm{Ni}$ spins are shown in (c) and (d) for the $a c$ and $a b$ planes, respectively. Note the different energy scales of panels (a) and (b) compared to (c) and (d). The energy with spins along the $a$ axis is taken as reference.

$D\left(\mathbf{S}_{\mathbf{i}} /\left|\mathbf{S}_{\mathbf{i}}\right| \cdot \mathbf{n}_{\mathbf{i}}\right)^{2}$, where $\mathbf{n}_{\mathbf{i}}$ is the site-dependent hard axis, described as $\mathbf{n}_{\mathbf{1}}=\mathbf{n}_{\mathbf{3}}=(\cos \alpha, 0, \sin \alpha), \mathbf{n}_{\mathbf{2}}=\mathbf{n}_{\mathbf{4}}=$ $(\cos \alpha, 0,-\sin \alpha)$ (see. Fig (1). According to the fitting, $\mathrm{D}=7.39 \mathrm{meV}$ and $\alpha=35.48^{\circ}$ : this implies the hard axis $\mathbf{n}_{\mathbf{i}}$ to be nearly along the $m 1$ bond, so that Co spins are aligned in an easy plane perpendicular to $\mathbf{n}_{\mathbf{i}}$. In terms of site-dependent anisotropy, we note that the stable AFM spin ordering $S_{1}=S_{2}=-S_{3}=-S_{4}$ is different from the orbital ordering $L_{1}=L_{3}, L_{2}=L_{4}$ which causes a local anisotropy. In such a configuration, all four spins are allowed to lie in the easy plane only when collinear AFM spins are pointing along the $b$ axis. Therefore the $b$ axis is the easy axis in the collinear AFM configurations for Co spins.

Our calculations show for $\mathrm{Ni}$ spins an opposite trend of $E_{S O C}$, with respect to the Co spin, both in the ac and $a b$ planes (cfr Fig. 2); moreover, the MAE is almost two orders of magnitude smaller, consistent with the spherical $d^{8}$ electronic cloud. Here the $b$ axis is the global hard axis.Assuming the easy axis to lie in the $a c$ plane and by fitting $E_{t o t}$ to the anisotropic term, we estimate $\mathrm{D}=-0.50 \mathrm{meV}$ and $\alpha=46.7^{\circ}$ Here $\alpha$ is close to $\pi / 4$ 


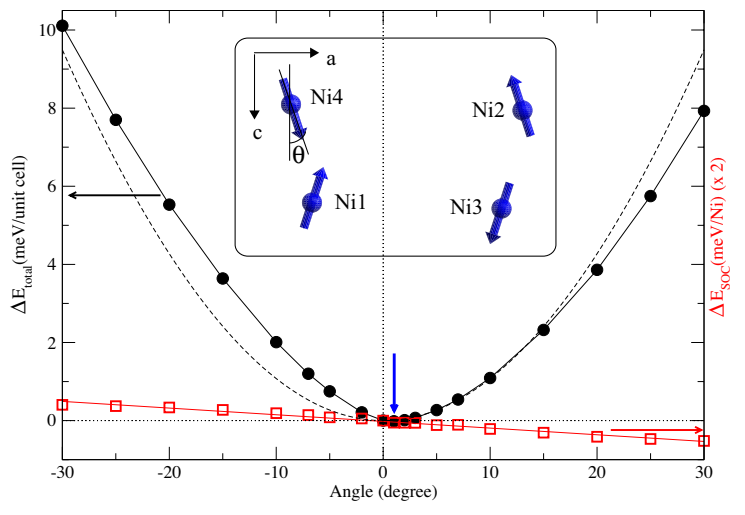

FIG. 3: Total energy $\Delta E_{\text {total }}$ (black solid line) and $\Delta E_{S O C}$ (dashed red line) at each $\mathrm{Ni}$ site vs the direction of noncollinear Ni spins in the "spin-cross" configuration (shown in the inset). The minimum is marked by a vertical blue arrow. The dashed line is a function proportional to $1-\cos \theta$, as a guide to the eye, to outline the "asymmetrical" behaviour of the DFT data.

so that two opposite contributions from local anisotropy nearly cancel out (Fig. 2 (c)) and induce a small in-plane anisotropy. The negative value of $D$ implies the existence of an easy axis $\mathbf{n}_{\mathbf{i}}^{\prime}$ of $\mathrm{Ni}$ spins in the $a c$ plane (see. Fig 1), $\mathbf{n}_{\mathbf{1}}^{\prime}=\mathbf{n}_{\mathbf{3}}^{\prime}=(-\sin \alpha, 0, \cos \alpha), \mathbf{n}_{\mathbf{2}}^{\prime}=\mathbf{n}_{\mathbf{4}}^{\prime}=(\sin \alpha, 0, \cos \alpha)$ which is close to the $\mathbf{n}_{\mathbf{i}}$ direction at Co spins. Therefore, the $c$ axis is the global easy axis for Ni spins if a collinear AFM configuration is assumed. However, in order to stabilize the local anisotropic term, the spins are expected to slightly tilt with respect to the $c$ axis. This deviation, denoted as "angled cross" spin configuration, has been already discussed by Chupis [9] in terms of Landau theory and suggested to play an important role for magnetoelectricity. This issue has been carefully investigated here by tilting the spins by an angle $\theta$ from the $c$ axis. Indeed, as shown in Fig. 3. the non-collinear spin structure in the $a c$ plane with $\theta \sim 1^{\circ}$ gives the lowest energy. We considered a generic Hamiltonian for $\mathbf{S}_{\mathbf{i}}(i=1$ to 4$)$ spins, including a Heisenberg term, an relativistic anisotropy term and a Zeeman-like term (see next paragraph, where a finite external $\mathrm{H}$-field will be introduced):

$$
\mathcal{H}=\sum_{\langle i, j\rangle} J_{i j} \frac{\mathbf{S}_{\mathbf{i}} \cdot \mathbf{S}_{\mathbf{j}}}{\left|\mathbf{S}_{\mathbf{i}}\right|\left|\mathbf{S}_{\mathbf{j}}\right|}+\sum_{i} D\left(\frac{\mathbf{S}_{\mathbf{i}}}{\left|\mathbf{S}_{\mathbf{i}}\right|} \cdot \mathbf{n}_{\mathbf{i}}\right)^{2}+\sum_{i} \mathbf{S}_{\mathbf{i}} \cdot \mathbf{H}
$$

In this $\mathrm{H}=0$ case, a delicate balance of the first two terms occurs: As the spin is tilted towards the local easy axis, the SOC-term is stabilized, whereas the $J_{i j}$ coupling becomes unstable. As a result, the equilibrium occurs in a non-collinear "angled-cross" spin-configuration.

Magnetoelectricity in $\mathrm{LiNiPO}_{4}$. The existence of a local magnetic anisotropy is particularly important in the context of magnetoelectricity. In order to investigate $\mathrm{ME}$ effects in $\mathrm{LiNiPO}_{4}$, hereafter we focus on the change in the spin configuration under a magnetic field with respect to the "angled cross" ground-state. The exchange coupling constants $J_{i j}$ were evaluated by total energy
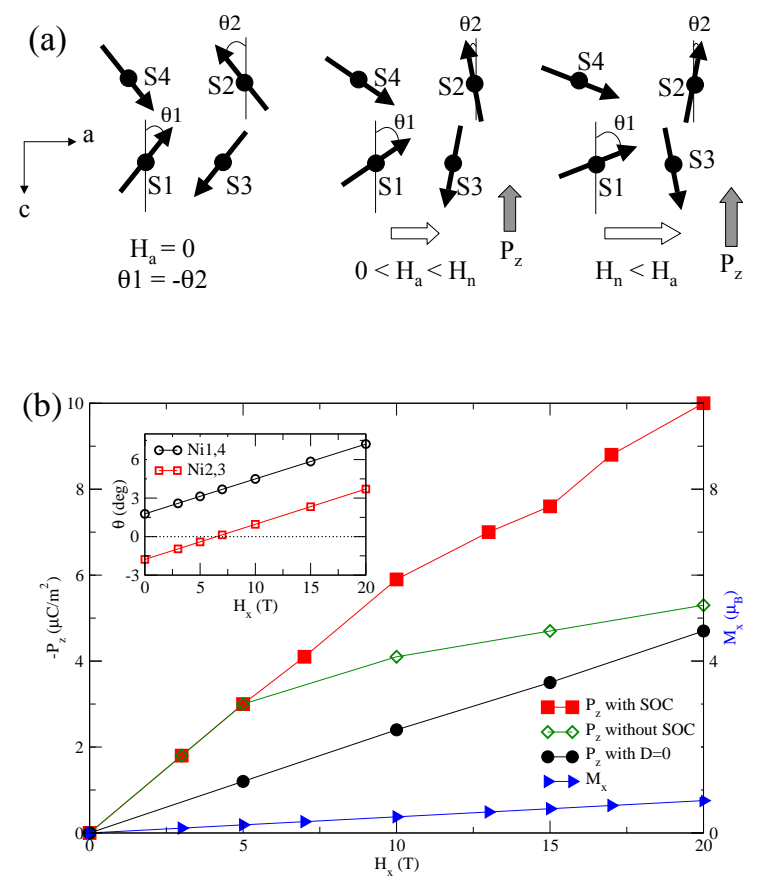

FIG. 4: (a) Spin configuration in the $a c$ plane under an applied field $H_{x}$. (b) Magnetic-field induced electric polarization along $z$ (with and without SOC, as well as considering $\mathrm{D}=0$ in Eq.(1), see text) in $\mathrm{LiNiPO}_{4}$. The magnetization $M_{x}$ is also shown (left-trianges, referred to the right $y$-axis) Inset: spin angle $\theta 1$ and $\theta 2$ (in degrees) vs $H_{x}(\mathrm{~T})$

differences fitted to Eq. (1), considering several AFM configurations. Our calculated values are $J_{12}=J_{34}=$ $-0.118 \mathrm{meV}, J_{13}=J_{24}=1.46 \mathrm{meV}, J_{14}=J_{23}=3.92 \mathrm{meV}$ (the positive sign means AFM coupling). The dominant $J_{14}$ keeps the spins in the AFM configuration, whereas $J_{12}$ and $J_{13}$ are responsible for removing the degeneracy of some AFM configurations 8], i.e. the AFM spin configuration $S_{1}=S_{2}=-S_{3}=-S_{4}$ gives the most stable energy. The $J$ values are consistent with the fact that four $\mathrm{Ni}$ sites are separated into two pairs (Ni1, Ni4) and (Ni2, $\mathrm{Ni3}$ ) by a Li intercalated layer, so that different pairs are expected to be weakly coupled. The anisotropy coefficient $D$ is also obtained from DFT, as reported above. A nonzero applied magnetic field, $H_{x}$, is expected to tilt the $\mathrm{Ni}$ spins from the angle-crossed spin configuration, with angles from the $c$ axis, denoted as $\theta_{1}$ and $\theta_{2} \cdot$.22] (Here we assume the relation $\theta_{1}=\theta_{4}$ and $\theta_{2}=\theta_{3}$ by considering the symmetry under the magnetic field.) By using DFT parameters, Eq.(1) is easily minimized by Newton method with $\theta_{1}$ and $\theta_{2}$ angles varying by few degrees in a way proportional to $H_{x}$ (cfr Fig目), inset).

Constraining the spin angles as obtained from the Newton minimization for each $\mathrm{H}$-field, we evaluate the electronic polarization. A small but finite $P_{z}$ is obtained, confirming magnetoelectricity. As shown in Fig. 4 all spins have positive $x$ components for $H_{x}>5 T$; however, the sign of the moment doesn't affect the induced $P_{z}$, 
which is rather linearly proportional to $H_{x}$, consistent with the experimentally observed behaviour at low temperature. From the slope of the $\mathrm{P}-\mathrm{H}$ curve from $\mathrm{H}=0$ to $10 \mathrm{~T}$, the linear ME coefficient $\alpha_{z x}$ is estimated as $0.59 \mu \mathrm{C} / \mathrm{m}^{2} \mathrm{~T}=0.74 \mathrm{ps} / \mathrm{m}$, comparable with the lowtemperature experimental value $\alpha_{z x}^{\exp }=1.5 \mathrm{ps} / \mathrm{m}$. [12]

Let us now investigate the microscopic origin of magnetoelectricity. Electric dipoles are found to be mainly induced by symmetric exchange 23], often referred to as "inverse" Goodenough-Kanamori (iGK) interaction 24] in Ni1-O-Ni4 (or Ni2-O-Ni3) bonds. For small $H$, the other conventional mechanism for magnetically-induced polarization - related to antisymmetric exchange and often labeled as "inverse Dzyaloshinskii-Moriya (iDM)" is negligible. However, for high magnetic fields, the iDM becomes sizeable (cfr Fig $4 \mathrm{~b}$ ), where the comparison between $P_{z}$ values including - or not - the SOC term is reported). In this context, we note that the existence of a local anisotropic term is important to induce $P$ : Assuming $D=0$ in Eq.(1), so that $\theta 1=\theta 2$, we find that the induced $P_{z}$ is less than half with respect to the nonzero $D$ case (cfr Fig $4 \mathrm{~b})$.

Finally, we estimate toroidal moments based on Ref. [5]. The collinear AFM spin configuration in $\mathrm{LiCoPO}_{4}$ induces $(0,0,-1.52) \mu_{B} \AA$, whereas the noncollinear spin configuration (at $\mathrm{H}=0 \mathrm{~T}$ ) in $\mathrm{LiNiPO}_{4}$ induces $(0.19,0.94$,
0) $\mu_{B} \AA$ per unit cell. We observe that the spin crossed state causes a small $T_{x}$-component; however the $T_{y}$ component, coming from the compensated AFM spin configuration, is relevant to the ME effect with finite $P_{z}$ and $H_{x}$.

In conclusion, we have presented a careful investigation of the spin-anisotropy in Li-based transition-metal phosphates. We found that $\mathrm{LiNiPO}_{4}$ shows a peculiar groundstate spin configuration: due to a site-dependent magnetic anisotropy, it shows non-collinear spins arranged as "angled-cross"-like. This is relevant in the context of magnetoelectricity: due to the ground-state noncollinearity, the spin configuration induced by an applied magnetic field leads to a net electric polarization, as shown by a realistic first-principles estimate of the magnetoelectric coefficient in agreement with experiments. Our results suggest a possible avenue for elucidating the origin of the ME effects in other compounds.

Acknowledgments. We thank Claude Ederer for helpful discussions. The research leading to these results has received funding from the European Research Council under the EU Seventh Framework Programme (FP7/20072013) / ERC grant agreement n. 203523. Computational support from Caspur Supercomputing Center (Rome) and Cineca Supercomputing Center (Bologna) is gratefully acknowledged.
[1] Amine, K; Yasuda, H; Yamachi, M, Electrochemical and Solid State Letters 3, 178 (2000).

[2] Le Bacq, O; Pasturel, A; Bengone, O, Phys. Rev. B, 69, 24 (2004).

[3] B. B. Van Aken, J. P. Rivera, H. Schmid and M. Fiebig, Nature 449702 (2008).

[4] Schmid, H. Ferroelectrics 252, 41 ?50 (2001).

[5] C. Ederer and N. A. Spaldin, Phys. Rev. B 76214404 (2007).

[6] N. A. Spaldin and M. Fiebig, Science 309, 391 (2005).

[7] Rev. Gen. Electr. 80143 (1971).

[8] D. Vakinin et. al., Phys. Rev. B 65224414 (2002).

[9] I. E. Chupis, Low Temperature Physics 26419 (2000).

[10] R. P. Santoro, R. E. Newnham and S. Nomura, J. Phys. Chem. Solids 27655 (1966).

[11] R. P. Santoro, D. J. Segal, and R. E. Newman, J. Phys. Chem. Solids 271192 (1966).

[12] T. B. S. Jensen, et. al., Phys. Rev. B 79, 092412 (2009).

[13] J.-P. Rivera, Ferroelectrics 161, 147 (1994).

[14] G.Kresse and J.Furthmüller, Phys.Rev.B 54, 11169 (1996).

[15] P. E. Blöchl, Phys. Rev. B 50, 17953 (1994).

[16] V.I.Anisimov, F.Aryasetiawan and A.I.Lichtenstein, J. Phys.: Cond. Mat. 9, 767 (1997).
[17] D. Vaknin,1 J. L. Zarestky, L. L. Miller, J.-P. Rivera and H. Schmid, Phys. Rev. B 65, 224414 (2002).

[18] I. Abrahams and K. S. Easson, Acta Cryst. C49, 925926. (1993).

[19] R.D.King-Smith and D.Vanderbilt, Phys. Rev. B 47, 1651 (1993); R. Resta, Rev. Mod. Phys 66, 899 (1994).

[20] J. Kübler, Theory of Itinerant Electron Magnetism (Oxford Science Publications, New York, 2000).

[21] H. J. Xiang, Su-Huai Wei, M.-H. Whangbo and Juarez L. F. Da Silva,Phys. Rev. Lett. 101, 037209 (2008).

[22] The effect of an applied magnetic field is taken into account only through local spin directions (i.e. neglecting dynamical effects by the field).

[23] S. Picozzi, I. A. Sergienko, K. Yamauchi, B. Sanyal and E. Dagotto, Phys. Rev. Lett. 77, 227201 (2007); S. Picozzi and C. Ederer, J. Phys. Cond. Matt. 21, 303201 (2009).

[24] K. Yamauchi, S. Picozzi, J. Phys. : Condens. Matter 21, 064203 (2009).

[25] H.Katsura, N.Nagaosa, A.V.Balatsky, Phys. Rev. Lett. 95, 057205 (2005); I.A.Sergienko et al. and E.Dagotto, Phys. Rev. B 73, 094434 (2006); M.Mostovoy, Phys. Rev. Lett. 96, 067601 (2006). 